\title{
INVERSION OF SIDE SCAN SONAR MOTION AND POSTURE IN SEABED GEOMORPHOLOGY
}

\author{
Weiliang Tao, Ph. D. \\ The School of Electronic Information, Wuhan University, Wuhan, Hubei, China \\ Yan Liu, Ph. D. \\ State Key Laboratory of Power Grid Environmental Protection, China Electric Power Research Institute, Wuhan, Hubei, China \\ Wenbin Hu, Ph. D. \\ The School of Electronic Information, Wuhan University, Wuhan, Hubei, China
}

\begin{abstract}
Side scan sonar measurement platform, affected by underwater environment and its own motion precision, inevitably has posture and motion disturbance, which greatly affects accuracy of geomorphic image formation. It is difficult to sensitively and accurately capture these underwater disturbances by relying on auxiliary navigation devices. In this paper, we propose a method to invert motion and posture information of the measurement platform by using the matching relation between the strip images. The inversion algorithm is the key link in the image mosaic frame of side scan sonar, and the acquired motion posture information can effectively improve seabed topography and plotting accuracy and stability. In this paper, we first analyze influence of platform motion and posture on side scan sonar mapping, and establish the correlation model between motion, posture information and strip image matching information. Then, based on the model, a reverse neural network is established. Based on input, output of neural network, design of and test data set, a motion posture inversion mechanism based on strip image matching information is established. Accuracy and validity of the algorithm are verified by the experimental results.
\end{abstract}

Keywords: side scan sonar; image matching; image fusion; neutral network; motion inversion

\section{INTRODUCTION}

Side scan sonar describes submarine topography in the form of grids and detects seabed morphology by recording and showing submarine backscatter echo of incident sound wave. Side scan sonar record captures a series of strip images without geographic azimuth information. Many difficulties need to be solved to form large-scale, high-precision, highresolution submarine landscape images. Image mosaic and geocoding can map the measured strip images to specified geographic coordinate system, thus synthesizing a whole regional geomorphic image. But existing processing means have difficulty to effectively eliminate image spots, stripe noise caused by noise, marine environment changes, insufficient navigation information accuracy, track bending, speed nonuniformity, unstable towfish posture as well as cracks and local distortion etc., caused by inaccurate image mosaic. These interference and distortion problems greatly hinder applications of automatic target detection, identification, seabed material classification, navigation.

In this context, we carried out research on side scan sonar image mosaic processing system, and established a new processing framework in order to improve quality of side scan sonar mosaic image and computational efficiency of corresponding operation. The processing model first filters spot noise and stripe noise of strip image obtained via side scan sonar. Then, adjacent strip image is matched with feature and region similarity metrics algorithm based on side scan sonar image characteristics. Relative position information of strip image is obtained by matching, so that 
track of side scan sonar carrier platform is inverted to solve the mispairing and mismatch problem. Finally, the matching strip image is merged and embedded to obtain the whole seabed geomorphology of the detection area.

This paper focuses on inversion of side scan sonar track in the whole frame. The random variation of navigational state of the carrier platform is the main interference that degrades accuracy of mosaic image, also the main source of mispairing and mismatch problem of strip image. As side scan sonar runs in the underwater environment, it is difficult for auxiliary navigation tool to locate it, and its accuracy and sensitivity cannot be guaranteed. It is a novel, intuitive and effective means to judge the misplaced points, correct the mismatched images and amend the location information by obtaining navigational status of side scan sonar platform. Based on the theory of artificial intelligence and pattern recognition, motion state information of the platform can be extracted by self-learning and generalization process from parameters such as translation, rotation and scaling obtained by multiple strip image matching analysis, and then motion trajectory of the platform can be calculated.

\section{RELATED WORKS}

With the deepening of requirements for underwater detection and ocean surveys, early extensive measurement and data processing methods are no longer applicable. Due to special working methods and poor working environment of sonar in ocean survey, relevant researchers at home and abroad have been carrying out a lot of research on motion error correction method of sonar platform and have made many achievements. There are different approaches for measurement of platform motion error according to sonar types.

The multi-beam sonar collects amplitude and phase information of multi-array elements. The data values of each measurement point are calculated by phase control of the above information, which has some anti-interference ability, and is less sensitive to motion disturbance of the platform. Yonggang Zou et al. analyzed existence of leakage detection area in the case of platform movement when measurement point distribution of multi-beam sonar measurement coverage area is uneven [1]. Fucheng Bai et al. carried out motion compensation of echo data received by multi-beam in the assumption that posture parameters of auxiliary sensor measurement are accurate [2]. Fanlin Yang analyzed relative positional relationship between posture sensor and transducer by multi-beam sonar measurement results of flat experimental waters, and thus eliminated offset error of posture sensor [3].

The processing of synthetic aperture sonar is based on linear uniformity of sonar platform, and the imaging results are greatly affected by motion error. Therefore, research on motion error correction of synthetic aperture sonar arouses wide concern of researchers. However, there is data redundancy in virtual aperture measurement of synthetic aperture sonar, which provides sufficient data support for inversion of motion trajectory of sonar platform. Peng Wu analyzed motion error model of synthetic aperture sonar, considering that MD (map drift) algorithm and COA (contrast optimization autofocus) algorithm can only compensate second-order phase error, and the resolution achievable is limited. The PGA (phase gradient autofocus) algorithm estimates the phase error directly from the echo signal. It can theoretically compensate phase error of any order. It has a wide application prospect in high-resolution imaging [4], but requires corresponding correction for strip mode. Dongsheng Chen et al., on the basis of DPC algorithm, relaxed limits of DPC algorithm on platform speed by calculating correlation of two-frame signal in "virtual phase center" [5]. Wei Liu et al. divided large aperture into a number of sub-apertures, estimated and compensated internal movement error of subaperture with the original echo data [6].

For the problem of motion error estimation and compensation of side scan sonar, there is no mature research reported in current literature. On the one hand, traditional side scan sonar is generally only used as an auxiliary means of measurement, which fails to undertake independent high-precision marine measurement tasks, so its accuracy requirement is not high; on the other hand, side scan sonar has not high data redundancy, and thus motion inversion and compensation are difficult for it compared to multi-beam sonar and synthetic aperture sonar. Weiqing Zhu et al. led the development of high-resolution sounding side scan sonar to solve sonar shortcomings in poor precision in area below the sonar, incapability to accurately distinguish submarine undulating area, shallow water area with interface impact and other areas with complex sound field, and thus high resolution of seabed topography and geomorphology can be obtained [7]. Multi-beam side scan sonar [8], multi-pulse side scan sonar can significantly improve mapping efficiency of submarine landform [9]. Interference side scan sonar [10] receives more phase diversity of sub-array receipt signal to obtain depth information of seabed features. Research on joint detection and even data, image fusion with side scan sonar and other ocean exploration systems such as multi-beam system has also achieved some success [11]. Side scan sonar systems are already capable of high-precision, high-resolution marine survey applications. In this context, it is of practical significance to study inversion and compensation of side scan sonar motion. Although data redundancy of side scan sonar is not high, there is a common coverage area in its strip image data, and regional pattern varies with the changes in motion and posture [12]. So the platform motion, posture change parameters can be calculated according to morphological parameters of the common coverage area.

In general, the current research on inversion and compensation of side scan sonar motion is of great significance to improve quality of side scan sonar images and expand its application in high-precision marine surveying and mapping $[13,14]$. It is a potential research direction to study trajectory self-correction method of side scan sonar platform under the condition of complex environment and unsteady platform. 


\section{EFFECT OF PLATFORM MOTION}

Disturbance of marine environment and human factors of ship handling platform will cause swing and translation of side scan sonar platform, which will cause blur and distortion of geomorphologic imaging of side scan sonar. Therefore, it is necessary to carry out motion compensation for it. The premise of compensation is capability to obtain motion state and posture parameters. As side scan sonar has towing navigation in the underwater, its motion state and posture parameters cannot be accurately obtained due to limitations of accuracy and sensitivity of navigation and positioning equipment, and even motion and posture measurement of the platform is ignored. In view of the above reasons, this paper studies motion state and posture parameters of the platform from echo data.

Firstly, the influence of platform motion on echo data is analyzed. Fig. 1 is the motion, posture error model which analyzes from six components of surge, sway, heaving, yawing, rolling and pitch.

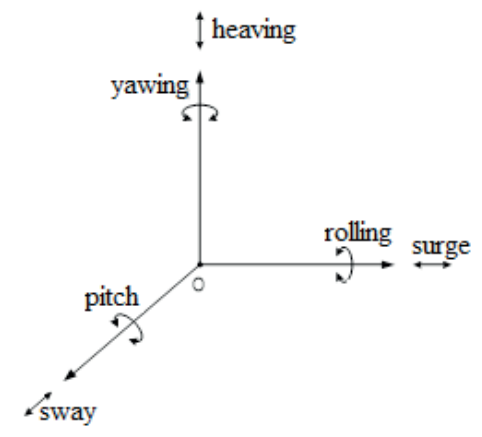

Fig. 1. Motion, posture error model of the platform

\section{INFLUENCE OF SURGE AND SWAY COMPONENTS ON SIDE SCAN SONAR STRIP IMAGE}

The effect of surge and sway on side scan sonar imaging is shown in Fig. 2(a) without considering other factors.
It can be seen that surge, sway only affects translational position of side scan sonar imaging area, without stretching, distortion and other effects. In the two measurement intervals, degree of surge, sway of the platform is relatively small, so its impact on quality of geomorphic map is relatively small.

The effect of surge and sway on strip image is represented by the corresponding translational displacement of the relative position of adjacent strip images.

\section{INFLUENCE OF HEAVING ON SIDE SCAN SONAR STRIP IMAGE}

The effect of heaving on side scan sonar imaging is shown in Fig. 2(b).

Obviously, platform heaving causes a greater impact on side scan sonar imaging. Under the influence of external conditions, sonar platform raises, which causes larger coverage area and larger seabed dot spacing between the two adjacent measure points. On the contrary, the coverage area becomes smaller and dot spacing between two adjacent measure points becomes smaller.

The effect of platform heaving on strip image is shown in scale of local position of adjacent strip image.

\section{INFLUENCE OF YAWING ON SIDE SCAN SONAR STRIP IMAGE}

The effect of platform yawing on strip image is shown in Fig. 2(c).

Ship's course changes of sonar platform or wind and waves will make the platform yaw. Yawing causes rotation in angle of sonar's acoustic beam coverage area. There is no significant changes in the coverage area sizes and measure points spacing between near and far beams.

Thus, yawing of sonar platform causes rotational change in adjacent strip images.

\section{INFLUENCE OF ROLLING ON SIDE SCAN SONAR} STRIP IMAGE

The effect of platform rolling on side scan sonar geomorphologic imaging is shown in Fig. 2(d).

As shown in the figure, rolling of sonar platform has a significant impact on side scan sonar geomorphologic imaging, which causes change in slant angle of sonar beam, so that actual irradiation area of the acoustic beam is translated, and dot spacing of near and far beam beams becomes larger or smaller. Rolling occurs frequently

Fig. 2. The effect of platform's motion on side scan sonar imaging 
in low-speed navigation, poor sea conditions, which greatly affects clarity of geomorphic images.

In summary, the effect of platform rolling on strip image is represented as translation and affine changes of adjacent strip images.

\section{INFLUENCE OF PITCH ON SIDE SCAN SONAR STRIP IMAGE}

The effect of platform pitch on side scan sonar geomorphologic imaging is shown in Fig. 2(e).

As shown in the figure, pitch of the sonar platform also has a significant impact on side scan sonar geomorphologic imaging. It causes actual irradiation area of the acoustic beam to be translated, and size of coverage area also changes, with measure points spacing getting larger or smaller. Pitch occurs when the sea condition is poor, which greatly affects clarity of geomorphic image.

The effect of platform pitch on strip image is represented as translation and affine changes of adjacent strip images.

From the above analysis, it can be seen that surge, sway, heaving, yawing, rolling and pitch of sonar platform causes translation, tilt and size change of sonar coverage area in geomorphologic imaging, resulting in random translation, rotation, scaling, affine change of adjacent strip images of side scan sonar. Image mosaics without image correction can cause blur, seams, discontinuities and distortions of side scan sonar image.

\section{INVERSION ALGORITHM FOR MOTION TRAJECTORY}

It can be seen that motion and posture changes of sonar platform are directly related to relationship between adjacent strip images of side scan sonar. It can also be said that scale, rotation and affine changes of strip images measured by side scan sonar reflect changes in motion and posture of sonar platform.

In measurement of strip image by side scan sonar, trajectory and posture changes of the platform will result in strip image coverage and proportional affine changes. Through the motion model of Section 3, we can establish the relationship between motion state of sonar platform and geomorphic strip image:

$$
\begin{gathered}
\mathbf{I}_{0}=\mathbf{Q} \mathbf{P}_{s} \cdot \mathbf{I}_{0} \\
\mathbf{I}_{m}=\mathbf{Q} \mathbf{P}_{m} \cdot \mathbf{I}_{0}
\end{gathered}
$$

Wherein, $\mathbf{I}_{0}$ is the real landform of the common measurement area of the source strip and matching strip; $\mathbf{I}_{s}$, $\mathbf{I}_{m}$ are geomorphic images of common measurement area of source strip and matching strip acquired by side scan sonar measurement, respectively. $\mathbf{Q}$ is sonar platform motion condition parameter matrix, which can convert submarine landform into a geomorphic strip image in a particular motion case; $\mathbf{P}_{s}, \mathbf{P}_{m}$ are position and posture parameter matrix of the sonar platform at measurement of source strip and matching strip by side scan sonar.

By the matching algorithm described above, the matching relationship between $\mathbf{I}_{s}$ and $\mathbf{I}_{m}$ can be obtained as follows:

$$
\mathbf{I}_{m}=\mathbf{R} \cdot \mathbf{I}_{s}
$$

Wherein, $\mathbf{R}$ is matching relationship matrix. That is, under the premise that rotation, scaling and affine changes of the two images are known, measurement point in source strip can be mapped to matching strip.

Simultaneous equation of $(1) \sim(2)$ can derive that:

$$
\mathbf{P}_{m}=\left(\mathbf{Q}^{-1} \mathbf{R Q}\right) \mathbf{P}_{s}
$$

Wherein, $\mathrm{Q}^{-1}$ is the inverse matrix of platform transformation matrix $\mathbf{Q}$.

Ground geomorphic data cannot be accurately measured and acquired, so motion state of the platform cannot be calculated by formulas (1) and (2). It can be seen from formula (4) that motion state of the sonar platform at measurement of matching strip can be derived from the motion parameters of sonar platform at measurement of source strip under the premise that transfer matrix $\mathbf{Q}$ and inverse matrix $\mathbf{Q}^{-1}$ of sonar platform motion parameters, and relative matching relationship $\mathbf{R}$ between source strip and matching strip are known.

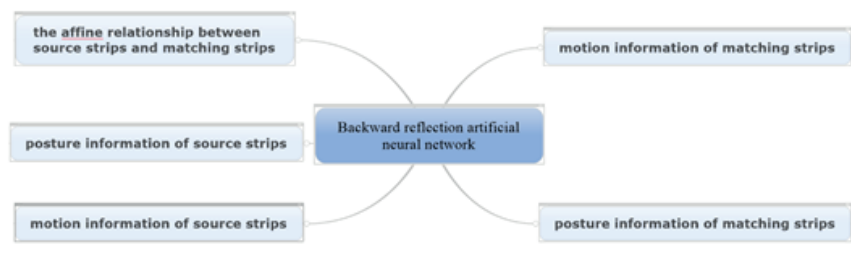

Fig. 3. The block diagram of inversion algorithm

\section{CONSTRUCTION OF NEURAL NETWORK}

There are many factors that cause changes in motion and posture of sonar platform and the platform often has various modes of motion such as surge, sway, heaving, yawing, rolling and pitch. It is very difficult to establish the model for inversion of sonar platform motion and posture via related parameters of adjacent strip images. But for the geometric model described in Section 3, it is easy to calculate region information and adjacent positional relationship of strip image from platform motion. In addition, in calculation of matching relationship of adjacent strip images according to matching point, it is necessary to assume that three or more matching points cause rigid change in the enclosed area, and this assumption will result in calculation error. Therefore, we consider application of artificial intelligence theory with good approximation ability and learning generalization ability for 
complex system, to achieve inversion operation of formula (4). As analyzed above, the block diagram of inversion algorithm is shown in Fig. 3.

Artificial neural network has good approximation ability for complex systems and can overcome influence of nonlinear factors in the system. Artificial neural network can simulate the relationship between multi-dimensional input and output without need to understand the complicated internal situation of the system, thus avoiding direct modelling of platform motion. In addition, artificial neural network construction is relatively simple, as there is no complex, time-consuming matrix operation. Based on the aforementioned functional model, a backward reflection artificial neural network structure is established, which is multi-layer sensor network structure and an error back propagation algorithm is for training. The system consists of input layer, output layer and hidden layer.

\section{a) Input layer}

The purpose of this network is to invert motion trajectory of the sonar platform from the matching relationship of adjacent strip images. The matching relation parameters of adjacent strip images can be obtained by the aforementioned matching algorithm as known input parameters. With platform motion position and posture parameters when the source strip image is measured as reference, the motion position and posture parameter of matching strip image are output.

After matching adjacent strip with image matching algorithm $[15,16]$, a number of matching point pairs are obtained for each pair of side scan sonar images. To determine the relative relationship between two images, more than three pairs of matching points are required. Since relative position information of matching point contains relative translation, rotation, affine relationship of adjacent strip image, it can be directly inputted as input information. To enhance algorithm effectiveness, relative position information of four pairs of matching points is used as input. According to equation (4), sonar platform motion information of source strip in the matching point is also input, and finally the sonar platform motion information is output when the four points in the target strip are measured.

\section{b) Hidden layer}

The neurons of the input layer weight the input feature parameters and send them to the hidden layer nodes. The hidden nodes sum the weighted values sent by the input nodes, and send them to the output layer after they are activated by activation function and weighted. Logistic function is selected as activation function:

$$
y_{k}=\frac{1}{1+\exp \left(-v_{k}\right)}
$$

Wherein, $v_{k}$ means the weighted sum of the input signals of $k$-th node of the hidden layer, $y_{k}$ means active output of $k$-th hidden layer node.
The activation function is non-linear, so that the network has nonlinear approximation ability.

\section{c) Output layer}

The output layer sums the weighted eigenvalues sent by the hidden layer and outputs it. In this paper, the output response is defined as surge, sway yawing, heaving rolling, yawing angle and pitch angle of the target strip.

\section{d) Learning algorithm}

The BP algorithm is used for neural network training, and the mapping problem of neural network learning input and output is transformed into a nonlinear optimization problem, wherein, the most popular and mature gradient descent algorithm in optimization is used. Network weight is corrected by iterative operation to minimize the cost of network output.

The cost function uses the mean square error between the neural network output and the desired output $d_{j}$ :

$$
E=\frac{1}{2} \sum_{j=1}^{N}\left(d_{j}-C_{j}\right)^{2}
$$

Wherein, $j$ is dimension number of output result of the output layer, $j=1,2, \cdots, N$. $C_{j}$ is $j$-th result of actual output of the neural network, $d_{j}$ is the desired output.

To sum up, the whole algorithm consists of forward calculation process and error back propagation process. The mean square error of the neural network is less than the specified value after sample set training, that is, the algorithm is considered as convergent.

In this paper, we set motion and posture parameters of reference strip and matching strip, calculate the coverage area of reference strip and matching strip using the geometric model described in the paper, and select a number of points from the two common overlapping regions as matching point pair. These parameters are used as the input and expected output of the neural network to train the network. After its convergence, it is considered that the network training is successful and can reflect mapping relation between matching-related information and motion parameters. The actual matching situation is input into the network, and then desired motion parameters can be obtained.

\section{TRAINING DATA PREPARATION}

When the network structure is established, the matching relationship between the adjacent strips and surge, sway, heaving, yawing angle, rolling angle and pitch angle information of the reference strip are input. Through training, learning and generalization of the network, navigation parameters of the strip to be tested can be obtained.

Training of the network requires a large amount of side scan sonar data as a basis. In the cases that data acquisition costs are high, experiment involves much equipment and the experimental process is complex, it is difficult to obtain 
enough information. Therefore, in the training phase, we consider auxiliary calculation of relevant parameters through modelling to obtain training data and meet needs for a large amount of training data at a small cost.

Using the model of Section 3, we can calculate the relevant parameters of adjacent strip images of the side scan sonar under the condition that the sonar platform has surge, sway, heaving, rolling and pitch motions. The relative position between the platform navigation trajectory and the matching point of reference strip of side scan sonar in the adjacent strip image is taken as the network input, and platform navigation trajectory of strip to be matched of side scan sonar is used as the expected output for network training.

\section{NEURAL NETWORK LEARNING}

Through the above modelling, calculation process, a large number of samples can be obtained. The samples are randomly divided into two subsets: training set and test set. Training subset is used to train neural network, adjust the network parameters so that physical relevance contained in the data can be learnt. And then system error is tested through the test set data. If the error range does not meet the requirements, then repeat the process, and continue to adjust the neural network until the system performance meets the requirements.

\section{NEURAL NETWORK GENERALIZATION}

After the end of the network learning, the system has the ability to extract regular output from the input data, that is, generalization ability. The platform navigation information of the reference strip is read from side scan sonar data, and matching of adjacent strip is calculated by matching algorithm. After organization, it is input into the trajectory inversion system and output of the system is regarded as trajectory of strip to be matched.

\section{EXPERIMENTS}

This section uses the experimental data generated by modelling to validate the motion inversion algorithm.

The existing side scan sonar navigation data lacks sufficient navigation information. In order to validate the inversion algorithm, we use the motion model of Section 3, assume that scheduled navigation speed, course of the platform and its height from the bottom are known when reference strip and matching strip are measured, and add $10 \%$ random posture and elevation changes on this basis, such as surge, sway, heaving, rolling and pitch and so on. It is easy to calculate geographical coordinates and overlapping area of each beam footprint in the strip image after knowing track, elevation and posture of both strips. A matching pair is randomly selected from the overlapping area, to replace 88 matching pairs of two strip images obtained by side scan sonar image matching algorithm based on SURF algorithm and typical region similarity calculation method. Every four matching points are randomly combined into one set, forming 64 sets of matching data. According to the model, platform position parameters and posture parameters (including instantaneous rolling angle, pitch angle and yawing angle of the platform) of each set of input data points of the source strip image are obtained. The matching point coordinate data of 64 sets of 32-dimensional data (4 pairs of matching points, a total of 8-dimensional data), reference platform position parameters (4-point geographical coordinates, elevation, a total of 12-dimensional data), reference platform posture parameters (4-point rolling angle, pitch angle and yawing angle, a total of 12-dimensional data) are input to the trained neural network, resulting in sonar platform position and posture parameters of 64 sets of matching strips at four matching points (including geographic coordinates, elevation, rolling angle, pitch angle and yawing angle, a total of 12-dimensional data). For all the matching points of the 64 sets, the repeated point trajectory parameters are averaged to obtain a unique coordinate.

Fig. 4 is the learning curve for training learning. After 80 cycles, the neural network learning performance is basically no longer improved. If the detection error of the system at this time is within the allowable range, it can be regarded that the network has passed training, and has established a model relationship between input and output.

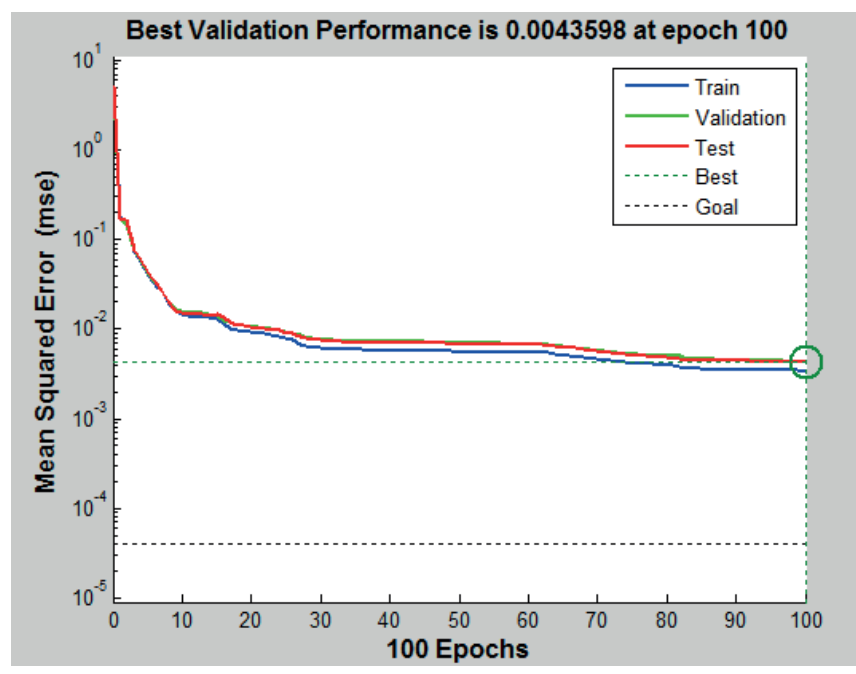

Fig. 4. Learning curve of training

The constructed neural network analyzes the input matching parameters and outputs the track coordinates and posture information of the sonar platform when it measures the matching strip. The track coordinates and elevation are compared with the coordinates calculated by the motion model, and the results are shown in Fig. 5. 


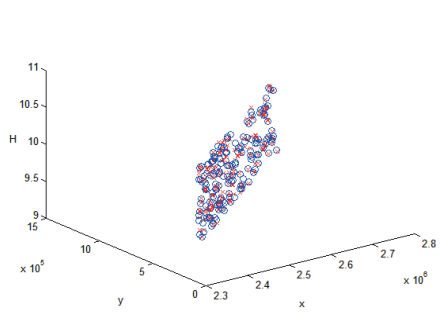

(a) Three-dimensional trajectory map Fig. 5. Platform trajectory obtar estimation results

As shown in Fig. 5, the red cross indicates the motion trajectory of the platform obtained by inversion algorithm. The blue circle represents the platform trajectory calculated by the model established in section 3. It can be seen that regardless of plane coordinates or elevation, the inversion results are basically able to reflect motion state of the platform.

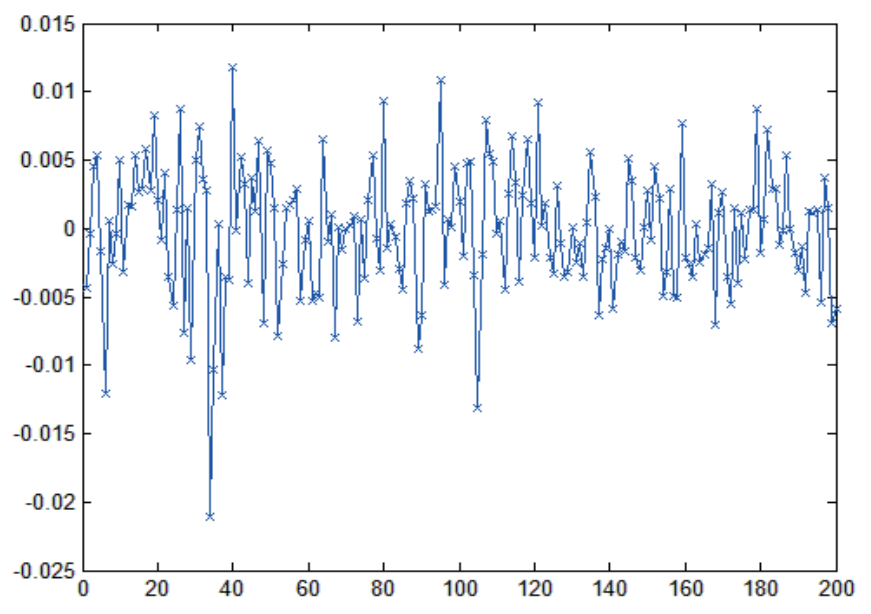

Fig. 6. The bias between the inversion result and the recorded coordinates

Fig. 6 shows the relative error percentage of the results of elevation inversion and the results of model calculation. It can be seen that the error is basically controlled within $2 \%$. The error levels of other matching indicators are shown in Table 1. As can be seen, the total error level meets the needs of the application.

Because platform motion is random, data transmission cannot be strictly synchronized, etc. the information provided by auxiliary navigation device can not reflect instantaneous fluctuation of its motion and posture, and the inversion algorithm proposed in this chapter can make up for the defect in this respect. It can be seen from the above experiment that the inversion algorithm has the ability to extract motion and posture parameters of the sonar platform accurately from the echo data, which provides the basis for the following tasks such as compensation, imaging and target recognition.
Tab.1. The error levels of matching indicators

\begin{tabular}{|c|c|c|c|c|c|c|}
\hline $\begin{array}{c}\text { Relative } \\
\text { error(\%) }\end{array}$ & $\begin{array}{c}\text { Coordinate } \\
\mathrm{X}\end{array}$ & $\begin{array}{c}\text { Coordinate } \\
\mathrm{X}\end{array}$ & Elevation & Rolling & Pitching & Yawing \\
\hline Minimum & -0.1 & -1.32 & -2.1 & -1.2 & -3.7 & -0.8 \\
\hline Maxmum & 0.986 & 1.7 & 1.18 & 2.8 & 1.2 & 3.3 \\
\hline
\end{tabular}

\section{CONCLUSIONS}

In this chapter, the motion trajectory inversion algorithm of side scan sonar platform based on artificial neural network is proposed. At the image level, motion parameters of the sonar platform are extracted from the measured echo data. The algorithm has the following advantages: a). Side scan sonar data redundancy is insufficient, so it is difficult to obtain its motion state with traditional methods. In this paper, motion parameters of matching strip are inverted with relative position relation of adjacent strip. b). Random motion of side scan sonar platform and its influence on strip data imaging are relatively complex and it is difficult to model motion parameters of inversion platform. The self-learning ability of artificial neural network algorithm can make the modelling process relatively simple and straightforward, and improve feasibility of the algorithm. c). The neural network algorithm requires use of a large number of samples for training, and it is difficult for usual ocean measurement task to meet the needs. The algorithm solves this difficulty by modelling calculation and construction of sufficient samples.

\section{BIBLIOGRAPHY}

1. Yonggang Zhou, Jingsheng Zhai, Yanchun Liu, Fuming Xiao, Juntao Jia: A bottom coverage estimation model based on trapezium for Multibeam System, Hydrographic Surveying And Charting, vol. 30, no. 2, pp. 15-18, 2010.

2. Fucheng Bai: The study on movement compensation new technique and hardware design of multi-beam swath bathymeter, Harbin Engineering University, Harbin, China, 2007.

3. Fanling Yang, Xiushan Lu, Jiabiao Li, Jinyun Guo: Correction of Imperfect Alignment of MRU for Multibeam Bathymetry Data, Geomatics and Information Science of Wuhan University, vol. 35, no. 7, pp. 816-820, 2010.

4. Peng Wu: Research on synthetic aperture sonar beamforming, Wuhan University of Technology, Wuhan, China, 2009.

5. Dongshen Chen, Haiting Yin, Jiyuan Liu, Shuqiu Li, Chunhua Zhang: A study on SAS movement compensation based on an improved DPC algorithm, Signal processing, vol. 23, no. 6, pp. 907-909, 2008. 
6. Wei Liu, Jiyuan Liu, Chunhua Zhang: Research on the combined SAS motion compensation method based on raw echo data and image data, Chinese journal of scientific instrument, vol. 4, no. 29, pp. 538-543, 2008.

7. Weiqing Zhu, Xiaodong Liu, Dongsheng Zhang, Fangsheng Zhang, Gaolin Qin, Zheng Liao, Changle Fang: High Resolution Bathymetric side scan Sonar, Ocean technology, vol. 24, pp. 29-35, 2006.

8. P. L Aa Gstad, P.E. Hagen, N. St O Rkersen, B. E. Marthinsen: Recent results from military operations with the HUGIN 1000 AUV, In: Proceeding UDT Europe 2005, Amsterdam, Nederland, 2005.

9. J. James: Sand wave morphology and development in the Outer Bristol Channel (OBel) Sands, Marine And Freshwater Behaviour And Physiology, pp. 163-170, 2008.

10. G. Mullins, J. Bird: 3D Sidescan with a Small Aperture: Imaging Microbialites at Pavilion Lake, Ocean 2007, Vancouver, BC, 2007.

11. Fanlin Yang, Ziyin Wu, Zhixing Du, Xianglong Jin: Co-registering and fusion of digital information of multibeam sonar and side-scan sonar, Geomatics and Information Science of Wuhan University, vol. 31, no. 8, pp. 740-743, 2006.

12. W. Tao, Y. Liu: Edge preserving filter of side scan sonar images with wavelet modulus maxima shift-correlative technique, International Journal of Imaging Systems and Technology, vol. 21, no. 4, pp. 349-355, 2011

13. J. M. Augustin, X. Lurton: Image amplitude calibration and processing for seafloor mapping sonars, Europe Oceans 2005, Brest, France, 2005.

14. L. Fonseca, C. Brown, B. Calder, L. Mayer, Y. Rzhanov: Angular range analysis of acoustic themes from Stanton Banks Ireland: A link between visual interpretation and multibeam echo sounder angular signatures, Applied Acoustics, vol. 70, no. 10, pp. 1298-1304, 2009.

15. W. Tao, J. Zhao, J. Liu, H. Zhang: Study on the Sidescan Sonar Image Matching Navigation Based on SURF, In: Electrical and Control Engineering (ICECE), 2010 International Conference, Wuhan, China, 2010.

16. Jianhu Zhao, Aixue Wang, Hongmei Zhang, Xiao Wang: Mosaic method of side-scan sonar strip images using corresponding features. IET Image Processing, vol. 7, no. 6, pp. 616-623, 2013.

\section{CONTACT WITH THE AUTHOR}

\author{
Weiliang Tao
}

e-mail:taowl2003@sina.com tel.: 18986199069

The School of Electronic Information Wuhan University Wuhan Hubei, 430074

\section{ChINA}

